\title{
Factors Affecting on Budget Utilization in Bahirdar City Administration Health Department, Ethiopia
}

\author{
Dr. Shahid Saheb, Dr. Ch. Venkata Krishna Reddy \\ Assistant Professor \\ Department of Public Financial Management and Accounting \\ College of Finance Management and Development, Ethiopian Civil Service University \\ Addis Ababa, Post Box No.5648, Ethiopia \\ Getachew Amsalu \\ Plan and Budget Coordinator \\ Ebinat Woreda Finance and Economic Development Office, South Gondar, Ethiopia
}

\begin{abstract}
The objective of this study was to investigate factors affecting budget utilization in case of Bahirdar City Administration Health Department. To achieve the objectives the researcher was adopted the mixed research approach. The study focused total count of all target population in the study area or census method used both quantitative and qualitative data, and it was collected through questionnaires of a total of 148 respondents. The Primary data was collected using closed ended questionnaires and prepared in the form of likert scale type by constructing into five-point scale. Independent variables are recoded in to three categories. To test the four research hypotheses which were closely associated with the research objectives, the research was used by Ordinal logistic regression model. The data was analyzed by using both descriptive and inferential statistics. The study found that the four independent variables (management support, organizational work culture, employee's related factor and monitoring \& evaluation) all have significant effects on budget utilization. Based on the findings, the study concluded that management support, organizational work culture, employee's related factor and monitoring \& evaluation significantly affects the budget utilization. The study recommended that budget utilization should be improved, give special attention to better budget plan forecasting and focus on performance of government budget. Keywords:Budget Utilization, Organizational Work Culture, Employee's Attitude, Management Support, Monitoring \& Evaluation, Health Department and Budget Performance.
\end{abstract}

DOI: $10.7176 / \mathrm{PPAR} / 9-8-04$

Publication date: August $31^{\text {st }} 2019$

\section{Introduction}

Effective and efficient budget utilization is determinant of socio-economic development of any given country including Ethiopia. In Ethiopia, resources like capital and recurrent budgets are major forms of financial planning in all regions. Most governmental policies that have the potential to make a difference to people's lives cannot to do so without a budget, so that a difficult aggregate expenditure control is highly required to assure an efficient use of resources in accordance with the government priorities(Allen and Tommasi 2001).

Besides to this, for effective use of budget and to gain an understanding of how public budgets have been utilized, and how they contribute to government policies, it is important to monitor the results of budget utilization. This led to the establishment of government monitoring and evaluation systems. It is important for governments to define and keep track of indicators to consider what they are trying to achieve with their policies and how far they are progressing and to use the information to plan accordingly. For this reason, there is a strong link between budget monitoring understanding how public budgets are being utilized (Rebecca, et,al. 2011).

Additionally, suggests to be taken the utilization of budget in case of Bahirdar City Administration Health Department. This study may be helps to contribute for efficient and effective on budget utilization in the future health sector for continuous improvement and sustainability of Bahirdar City Administration health department. This motivates the researcher to study factors affecting budget utilization.

\section{Statement of problems}

Both the developed and developing countries are mainly focusing about budget utilization for the development and its existence. Thus, efficient utilization of government budget is the primarily focus for the achievement of intended goals and another aspects about effective utilization of budget are needed. Therefore, different levels of Governments are concentrating to preparation and utilization of budget for the fulfillment of their requirements like recurrent and capital aspects. Moreover, most of the government policies that have the potential to make their difference levels of peoples' standard of living and sustainability of society cannot be achieved without efficient and effective utilization of government budget. Government assign high budget for health sectors, but the budget faces over and underutilization. Specially, this problem occurred in BDCAHD. 
The Ethiopian budget reform practiced by existing government, decentralized the authority to prepare and utilize budget, and to manage expenditure was from Federal, Regional, Zonal and Woreda levels. To enhance quality of health services, it is mandatory to make sure that the existing financial resources should be utilized properly in health department and included in health centers. In this context some of the researchers are focused to solve the research problems relevant to budget preparation, utilization and control are specified below.

Tilahun Bogale (2010) studied budget management and control special emphasis on ministry of national defense, his study showed only to obtain, assess, and analyze publicly available data on the budget management and control of Ethiopian Defense Ministry.

Another study by Ketema Muluneh,(2015) examined the assessment of budget preparation and utilization in case of Addis Ababa City Administration Health Bureau and revealed that there is no accountability in budget because, lack of adequate and experienced expert in the budget department.

Another study by Ashebir Regassa,(2016) on public expenditure management with an emphasis on resource utilization, a case of Benishangul Gumuz Regional State. As Ashebir has mentioned his study as the statement of the problem, developing nations are characterized by poor resource utilization. Moreover, the needs of citizens' development, quality service and growing expenditure have become barrier in the need of more resource. As a result, poor expenditure management leads the Benishangul Gumuz Regional State to ineffective resource utilization of scarce resources.

Another study by Geletaw Demera (2017) studied that determinants of budget control in public organization in case of Benishangul Gumiz Regional State and exploiting opportunities which factors had the most effect on budget control. Specifically, how budget planning process, management support, competent internal audit staff, organizational commitment, budget monitoring and evaluation, information and communication and cost reduction influence public organizations budget control were examined and answered which used to know the remedial action public organization take overcome lack of internal control.

Another study by Mulugeta Dassa (2017) on assessment of public budget allocation practices in the case of BOFED of the SNNPRS. Mulugeta has mentioned in his study as focused to find out the solutions of some research problem that is participation and transparency in the budget allocation practices of SNNPRS, BOFED.

However, in the above five researcher most studies focus on related in assessment of budget preparation and utilization, one researcher Geletaw Demera (2017) conducted research on determinants of budget control. Therefore, the gaps of the research factors are not hypothesized over and under budget utilization. So this study concentrate on the factors affecting budget utilization in case of BDCAHD, selectively focused on four factors this factors are management support, organizational work culture, employees related factor and monitoring \& evaluation.

\section{Research Gap}

The entire empirical studied Ethiopian contexts listed above are focused on budget related studies in different parts of Ethiopia, but the study was not conducted on factors affecting budget utilization in case of BDCAHD. So this study found this gap and tried to fill the gap by conducting the study.

\section{Literature review}

Budgeting is defined as a form of financial planning and source budget is utilized to impose the strategy of a country. Consequently, a budget is composing of different functional budget that could help a country for such development such as agriculture, technology, tourism, and other budget (Smith and Megeary1997). Budget is to be considered as financial plans. As this time the term budget is used to mean a plan for financing government during a definite period. The components of budget process include preparation, planning, utilization, accounting, control, reporting, monitoring \& evaluation as well as the existing legal frameworks. Budget is used as performance evaluation tools. Budgets are therefore merely a collection of plans and forecasts. Budget is that means of allocation and utilization of various resources to different sectors and agencies over a given period of time usually one year. Budgets are cost control devices which aimed at keeping cost within certain specified limits and revenue at expected levels to achieve the economic plans set and social policies been pursued. Budgets are future project financial performance which enables evaluating the financial viability of a chosen strategy. In most organizations this procedure is formal by making annual budgets and checking performance alongside budgets (Silva and Jayamaha,2012).

Budget utilization is take place throughout financial year and it's critical for any institution to perform. Public expenditure policy and the manner in which are managed would impact on budget utilization. It is the actual execution of the budget and application of funds to the planned activities (Kirira, 2007). There should be distribution of responsibilities for budget utilization, budget appropriation management rules and budget revisions, various special issues related to budget utilization, and the monitoring of budget execution. At the same time budget execution covers both activities related to the implementation of policies and tasks related to the administration of the budget. The central agencies and the spending agencies are involved in these tasks. The 
distribution of responsibilities in budget management should be organized according to the agencies' respective areas of responsibility and accountability (Tommasi, 2013).

\section{Objectives of the study}

i. General objective of the study

The main objective of this study is to investigate the factors affecting budget utilization in case of BDCAHD.

ii. Specific objectives of the study

The study has attempted to address the following specific objectives:

- To examine the contribution of management support for budget utilization in case of BDCAHD.

- To identify the effect of organization work culture on budget utilization in case of BDCAHD.

- To assess the employees related factors effect on budget utilization in case of BDCAHD.

- To establish the effect of monitoring and evaluation on budget utilization in case of BDCAHD.

- To provide necessary recommendation based on the results of the study.

\section{Research Hypothesis}

The study employee the hypotheses, these hypotheses are predictions about the outcome of the results, and they may be written as alternative hypotheses specifying the results to be expected. They also may be stated in the null form, indicating no expected difference or no relationship between groups on a dependent variable (Creswell, 2009). Therefore, in order to verify the relationship between dependent variable and independent variables to accept or reject the significance of the study, the following hypotheses were developed regarding factors affecting budget utilization in case of BDCAHD.

$\mathbf{H}_{1}$ : MS has significant effect on budget utilization in case of BDCAHD.

$\mathbf{H}_{2}$ : OWC has significant effect on budget utilization in case of BDCAHD.

$\mathbf{H}_{3}$ : ERF has significant effect on budget utilization in case of BDCAHD.

$\mathbf{H}_{4}$ : M\&E have significant effect on budget utilization in case of BDCAHD.

\section{Methodology}

To carry out the research objectives of this study were used descriptive research design using an inferential and descriptive statistics, this study involving both quantitative and qualitative research approaches (mixed approaches). The study was to be collected by using structured questionnaires and interviews. The total target population was 148 respondents used to Census method, both primary and secondary data collection methods are used. The responses presented in a five-point likert scales. The dependent variable (budget utilization) a total of 7 closed ended questions were developed to measure five point Likert scale method of budget utilization. In this study, there were four dimensions of independent variables composed of this study considered were management support, organizational work culture, employees, related factor and monitoring \& evaluation are different dimension of budget utilization factors. A total of 24 closed ended items was designed to 6 items for all each four independent variables were raised with the overall budget utilization effect. Thus, the respondents were requested to select their own choice based on five point likert scale method, and this data was recode to 3 groups that means strongly disagree code 1 and disagree code 2 are considered recoded as a1, neutral code 3 are recoded as a 2 and also agree code 4 and strongly agree code 5 are used as recoded a 3.

\section{Results and Discussions}

\subsection{Response Rate}

During the survey, a questionnaire was distributed to a total of 148 participants who are found in BDCAHD staffs included ten health centers and finance department staffs. From the total of 148 questionnaires distributed, 144 usable questionnaires were collected with 97.3 percent response rate. The percentage shows greater response rate of the research study conducted by the researcher, so the analysis was made based on 144 responded questionnaires.

\subsection{Reliability analysis}

To measure the consistency of the questionnaire particularly the likert-type scale, the reliability analysis is essential in reflecting the overall reliability of measuring. To carry out the reliability analysis, Cronbach's alpha test is the most common measure of scale reliability and a value greater than 0.7 is very acceptable (Field 2009, Cohen and Sayag 2010). 
Table 1: Reliability Statistics

\begin{tabular}{ccc}
\hline \hline Cronbach's Alpha & $\begin{array}{c}\text { Cronbach's Alpha Based on } \\
\text { Standardized Items }\end{array}$ & N of Items \\
\hline .836 & .839 & 31 \\
\hline \hline
\end{tabular}

Source: field survey 2018, output generated by SPSS

All 31 items of dependent and independent variables returned Cronbach's alpha value is 0.836 which is greater than 0.7 , then the responses generated for all variables used in this research were reliable enough for data analysis.

\subsection{Validity analysis}

In this study, to determine the validity of instrument, a pilot test has been conducted on $10 \%$ of the main sample 15 questionnaire was made by the researcher was used validity measurement for this study. Additionally, the improved version of the questionnaire was printed, duplicated, and dispatched to the sample, respondents may have varying capacities for being able to complete written questionnaires by English language, due to that the questionnaire was translated into Amharic version (Ethiopian national language).

\subsection{Demography Characteristics of Respondents}

Table 2: Background Profile of the Respondents

\begin{tabular}{|c|c|c|c|}
\hline Items & Options & Frequency & Percent \\
\hline \multirow{3}{*}{ Gender } & Female & 78 & 54.2 \\
\hline & Male & 66 & 45.8 \\
\hline & Total & 144 & 100 \\
\hline \multirow{4}{*}{ Age of respondent } & $18-30$ & 56 & 38.9 \\
\hline & $31-45$ & 69 & 47.9 \\
\hline & $46-60$ & 19 & 13.2 \\
\hline & Total & 144 & 100.0 \\
\hline \multirow{6}{*}{ Level of education } & Below certificate & 4 & 2.8 \\
\hline & Certificate & 12 & 8.3 \\
\hline & Diploma & 58 & 40.3 \\
\hline & First degree & 66 & 45.8 \\
\hline & Second degree and above & 4 & 2.8 \\
\hline & Total & 144 & 100.0 \\
\hline \multirow{5}{*}{$\begin{array}{l}\text { Year of work experience } \\
\text { (service) }\end{array}$} & $\leq 5$ years & 28 & 19.4 \\
\hline & 6 to 10 years & 66 & 45.8 \\
\hline & 11 to 15 years & 28 & 19.4 \\
\hline & Above 15 years & 22 & 15.3 \\
\hline & Total & 144 & 100.0 \\
\hline \multirow{9}{*}{ Position or status in the organization } & Leaders and management committee & 37 & 25.7 \\
\hline & Finance officer & 20 & 13.9 \\
\hline & Budget and plan officer & 5 & 3.5 \\
\hline & Auditors & 3 & 2.1 \\
\hline & Purchasers & 16 & 11.1 \\
\hline & HIT & 11 & 7.6 \\
\hline & Cashier & 48 & 33.3 \\
\hline & Store management officer & 4 & 2.8 \\
\hline & Total & 144 & 100.0 \\
\hline
\end{tabular}

\section{Source: field survey (2018)}

\subsection{Correlation analysis}

Spearman correlation was computed; because of categorical data. Interpretations are based on Joe W. kotrlik; J, C., Atherton; A. Williams and M. Khata Jabor (2011) way of determining the strength of the relationship. Accordingly, if $r=0.01$ up to 0.09 the strength of correlation said to be negligible association; if $r=0.10$ up to 0.29 low association; if $r=0.30$ up to 0.49 moderate association; if $r=0.50$ up to 0.69 substantial or significant association and if $\mathrm{r}=0.70$ and above very strong association. 
Table 3: Correlations

\begin{tabular}{|c|c|c|c|c|c|c|c|}
\hline & & & $\mathrm{MS}$ & $\mathrm{OWC}$ & ERF & $\mathrm{ME}$ & $\mathrm{BU}$ \\
\hline \multirow{10}{*}{ 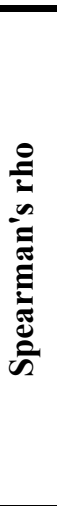 } & MS & Correlation Coefficient & 1.00 & & & & \\
\hline & & Sig. (2-tailed) & $\cdot$ & & & & \\
\hline & OWC & Correlation Coefficient & $.540^{* *}$ & 1.00 & & & \\
\hline & & Sig. (2-tailed) & .000 &. & & & \\
\hline & ERF & Correlation Coefficient & $.470^{* *}$ & $.504^{* *}$ & 1.00 & & \\
\hline & & Sig. (2-tailed) & .000 & .000 & . & & \\
\hline & $\overline{\mathrm{ME}}$ & Correlation Coefficient & $.472^{* *}$ & $.488^{* *}$ & $.443^{* *}$ & 1.00 & \\
\hline & & Sig. (2-tailed) & .000 & .000 & .000 & . & \\
\hline & $\mathrm{BU}$ & Correlation Coefficient & $.555^{* *}$ & $.562^{* *}$ & $.535^{* *}$ & $.502^{* *}$ & 1.00 \\
\hline & & Sig. (2-tailed) & .000 & .000 & .000 & .000 & \\
\hline
\end{tabular}

**. Correlation is significant at the 0.01 level (2-tailed).

Source: field survey 2018, output generated by SPSS

\subsubsection{Correlation between management support and BU}

From the Spearman correlation analysis result on table 3 above, management support is positively and significantly related to budget utilization $(\mathrm{r}=.555 * *$ and $\mathrm{p}=.000)$. This indicates that when management support increases, budget utilization can also be increase and vice versa. The correlation value shows the relationship between management support and budget utilization is substantial or significant association.

\subsubsection{Correlation between organizational work culture and BU}

As indicated in table 3 above, organizational work culture is positively and significantly related to budget utilization $(\mathrm{r}=.562 * *$ and $\mathrm{p}=.000)$; when organizational work culture increases, budget utilization can also be increase and vice versa and the relationship between them is substantial or significant association.

\subsubsection{Correlation between employee related factor and $\mathrm{BU}$}

The spearman correlation analysis result indicated table 3 above also shows employees related factor is positively and significantly related to budget utilization $\left(\mathrm{r}=.535^{* *}\right.$ and $\left.\mathrm{p}=.000\right)$. The correlation value indicates the relationship between employee's related factor and budget utilization is substantial or significant association.

\subsubsection{Correlation between monitoring $\&$ evaluation and $B U$}

Table 3 also revealed that monitoring \& evaluation is positively and significantly related to budget utilization(r $=.502 * *$ and $\mathrm{p}=.000$ ). The relationship between monitoring \& evaluation and budget utilization is also substantial or significant association.

From spearman correlation analysis result, four independent variables have a positive and significant relationship with the dependent variable of budget utilization in BDCAHD, they are statistically significant at $99 \%$ confidence level or correlation is significant at the $1 \%$ or 0.01 levels.

Table 4. Summary of correlation

\begin{tabular}{|c|c|c|c|}
\hline Independent variables & $\begin{array}{c}\text { Correlation } \\
\text { coefficient }\end{array}$ & p-value & $\begin{array}{c}\text { Relation between } \\
\text { DV \& IV }\end{array}$ \\
\hline Management support & 0.555 & 0.000 & Positively \& significantly \\
\hline Organizational work culture & 0.562 & 0.000 & Positively \& significantly \\
\hline Employees related factor & 0.535 & 0.000 & Positively \& significantly \\
\hline Monitoring and evaluation & 0.502 & 0.000 & Positively \& significantly \\
\hline
\end{tabular}

\section{Source: Output generated by SPSS (2018)}

\subsection{Diagnostic test for Ordinal logistic regression model analysis}

Ordinal logistic regression models are logistic regressions that model the change among several ordered values as a function of each unit increased by the predictor. Ordinal logistic regression (ordinal regression) is used to predict an ordinal dependent variable given one or more independent variables. In this ordinal logistic regression model there are four assumptions, in these assumption listed in chapter three.

8.6.1. Test of model adequacy

Ordinal logistic regression was a type of logistic regression analysis that when the response variable is categorized more than two with having natural order or rank. That is, we can rank the values, but the real distance between categories is unknown. Under ordinal logistic regression analysis we can deal model fitting information, goodnessof-fit, pseudo R-square, parameter estimates of odd ratios and test of parallel lines. This study was used in logit link function.

Logit link function was used in the analysis because it is evenly distributed categories and reasonable choices 
when the changes in the cumulative probabilities are gradual and logit involves all levels of the response and dichotomizes the response scale.

$\mathrm{R}$-squared measures the proportion of variation in the dependent variable that is explained by the independent variables (or by the ordinal logistic regression model). It is a goodness-of-fit statistic. Its value bounded between zero and one (inclusive), that is, the largest value that R-squared can assume is one or $100 \%$ (in which case all observations fall on the regression line, plane or surface), and the smallest it can assume is zero. In model fit information the $\mathrm{p}$-value is less than $5 \%$, the final model gives a significant improvement over the base line intercept only model.

\subsubsection{Evaluate the regression model (measuring strength of association)}

There are several-like statistics that can be used to measure the strength of the association between the dependent variable and independent variables.

In the nonlinear regression model, the coefficient of determination R-square summarizes the proportion of variance in the dependent variable associated with the predictor (independent variables, with larger R-square values indicating that more of the variation is explained by the model.

The following methods are used to estimate the coefficient of determination. Cox and Snell (1989) R-square is based on the log likelihood for the model compared to the log likelihood for a baseline model. However, with categorical outcomes, it has a theoretical maximum value of less than 1, even for a model. Nagelkerke (1991) Rsquare is an adjusted version of the Cox \& Snell that adjusts the scale of the statistic to cover the full range from 0 to 1 . McFadden (1974) R-square is another version, based on the log-likelihood kernels for the intercept-only model and the full estimated model. The model with the largest R-square statistic is "best" according to this measure. "perfect". In this study of ordinal logistic regression model the researcher used Nagelkerke R square because of the above reasons.

Table 5: Pseudo R-Square

\begin{tabular}{ll}
\hline \hline Cox and Snell & .489 \\
Nagelkerke & .545 \\
McFadden & .295 \\
\hline \hline
\end{tabular}

Link function: Logit.

Where:- R-squared is the proportion of variation in the dependent variable is explained by independent variables.

The overall enter method of ordinal logistic regression model summarized in the table 5 above, indicates that Rsquared (Nagelkerke) is 0.545 or $54.5 \%$. Therefore, as it indicated that pseudo R-squared is the proportion of variation in the dependent variable of budget utilization that is explained by the four independent variables. Therefore, it is pointed out that 54.5 percent of factors affecting budget utilization (management support, organizational work culture, employees' related factor and monitoring \& evaluation) have the power to explain the dependent variable of budget utilization in BDCAHD. The remaining 0.455 or $45.5 \%$ of the variance was affected or explained by other variables not included in this study and other variables affect budget utilization which is out of this study.

\subsubsection{Model fitting information}

Before start looking at the individual predictors in the model, the researcher needs to find out if the model gives adequate predictions. The model fitting information table 6 , below shows that the $-2 \log$ likelihood for the intercept only and final models can be used in comparisons of nested models. The statistically significant chi-square statistic $(\mathrm{p}$-value $=0.00<0.05)$ indicates that the final model gives a significant improvement over the baseline intercept only model.

Table 6: Model Fitting Information

\begin{tabular}{lrrrr}
\hline \hline Model & $\mathbf{- 2}$ Log Likelihood & Chi-Square & Df & Sig. \\
\hline Intercept Only & 199.309 & & & \\
Final & 102.705 & 96.604 & 8 & .000 \\
\hline \hline
\end{tabular}

\section{Link function: Logit.}

The test for significance of the model stated earlier that the adequacy of the ordinal logistic regression model is equivalent to testing the hypothesis:

$\mathbf{H}_{0}: \boldsymbol{\beta}_{2}=\boldsymbol{\beta}_{3}=\ldots=\boldsymbol{\beta}_{\mathrm{k}}=\mathbf{0}$

$H_{1}: H_{0}$ is not true (at least one $\beta \mathbf{j} \neq 0$ ), sig-value $=0.000$

$\mathrm{H}_{0}$ : states that all regression coefficients are insignificant (or none of them explains the dependent variable).

Failing to reject the null hypothesis means that such model is inadequate.

The chi-square test is designed to test the significance of all variables or set of variables in a regression model. The significant chi-square statistics indicates that the final model gives a significant improvement over the baseline intercept - only model. This basically tells us that the model gives better predictions than if we just guessed based on the marginal probabilities for the outcome categories. 
Considering the SPSS output in table 6, above show that the chi-square-statistics is 96.604 and the sig. value is 0.000 less than $0.01(1 \%)$. Thus we reject the null hypothesis, there is no significant non-linear relationship between the dependent variable and the independent variables at the $99 \%$ of confidence interval or $1 \%$ significance level of $\alpha$ and this conclude that there is a significant relationship between budget utilization and at least one of the independent variable included in the regression model.

\subsubsection{Goodness of fit}

Goodness of fit measures large observed significance levels. The goodness of fit is given in table 7 below, this table contains Pearson chi-square statistic for the model and another chi-square statistic based on the deviance. These statistics are intended to test whether the observed data are inconsistent with the fitted model. If they are not the significance values are large then we would conclude that the data and the model predictions are similar and that you have a good model. The large value for significant shows we have a good model.

$\mathbf{H}_{\mathbf{0}}$ : The model is a good fit (adequate)

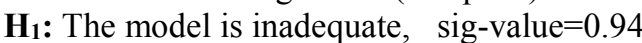

The table 7 below shows the goodness of fit for the ordinal logistic regression model. The Pearson sig- value is therefore 0.94 or 94.0 percent. Therefore the result, shows that the analysis of the model does fit very well (sig. value $0.94>0.05)$, that is fail to reject the null hypothesis depending on the observed data also the model is good fit. Decision do not reject the null hypothesis of the model is a good fit and conclusion the non-linear ordinal logistic regression model set earlier is a good fit model. Goodness-of-fit measures have large observed significance levels, so it looks that the model fits.

Table 7: Goodness-of-Fit statistic

\begin{tabular}{lccr}
\hline \hline & Chi-Square & Df & Sig. \\
\hline Pearson & 86.137 & 108 & .940 \\
Deviance & 68.621 & 108 & .999 \\
\hline \hline
\end{tabular}

Link function: Logit.

\subsubsection{Test of parallel line}

One of the assumptions underlying ordinal logistic regression is that the relationships between each pair of outcome groups are the same. This is commonly referred to as test of parallel lines. Test of parallel line is referred to as lines because the null hypothesis states the slope coefficients in the model are the same across response categories and lines of the same slope are parallel.

When model fit of an ordinal regression assume that the relationship between the independent variables and the dependent variable are the same for all the logits. That means the results are a set of parallel lines, one for each category of the outcome variable. Check this assumption by allowing the coefficients to vary, estimating them, and then testing whether they are all equal. The result of the test of parallelism is in table 8 below, the row labeled null hypothesis contains $-2 \log$ likelihood (-2LL) for the constrained model, the model that assumes the lines are parallel. The row labeled general is for the model with separate lines. You want to know whether the general model results in a sizeable improvement in fit from the null hypothesis model. The entry labeled chi-square is the difference between the two -2 log-likelihood values (null hypothesis minus general). If the lines or planes are parallel, the observed significance level for the change should be large, since the general model does not improve the fit very much. The parallel model is adequate. You do not want to reject the null hypothesis that the lines are parallel. If you do reject the null hypothesis, it is possible that the relationship between the independent variables and dependent variable are not the same for all logits.

Table 8: Test of Parallel Lines

\begin{tabular}{lcccc}
\hline \hline Model & -2 Log Likelihood & Chi-Square & Df & Sig. \\
\hline Null Hypothesis & 102.705 & & & \\
General & $71.928^{\mathrm{b}}$ & $30.777^{\mathrm{c}}$ & 24 & .160 \\
\hline \hline
\end{tabular}

Link function: Logit.

$\mathrm{H}_{\mathrm{o}}$ : The location parameters (slope coefficients) are the same across response categories.

$\mathrm{H}_{1}$ : The null hypothesis is not true, Sig-value $=0.160$

If we were to reject the null hypothesis based on the significance of the Chi-Square statistic, we would conclude that ordered logit coefficients are not equal across the levels of the outcome. If we fail to reject the null hypothesis, we conclude that the assumption holds. Therefore as it shown in the above table sig- value is 0.160

Decision: do not reject the null hypothesis.

Conclusion: the location parameters (slope coefficients) are the same across response categories.

\subsubsection{Multicollinearity test}

Multicollinearity testing can be done by looking at value of Variance Inflation Factors (VIF) and tolerance factors (1/VIF). The VIF was used to check for cases of multicollinearity among the independent variables.

Gashayie (2013) indicates that multicollinearity is a violation that no independent variables are nearly or 
highly correlated, as a result high correlation among independent variables will makes hard to separate the effects of individual variables.

The severities of the problem in multicollinearity across the independent variables are detected through VIF. According to (Gujirati, 2004), as a rule of thumb variables are considered as highly collinear if the VIF exceeds 10.

Table 9: Multicollinearity Coefficients

\begin{tabular}{|c|c|c|}
\hline \multirow[b]{2}{*}{ Independent variables } & \multicolumn{2}{|c|}{ Collinearity Statistics } \\
\hline & Tolerance & VIF \\
\hline Management support & .591 & 1.692 \\
\hline Organizational work culture & .537 & 1.861 \\
\hline Employees related factor & .646 & 1.547 \\
\hline $\begin{array}{r}\text { Monitoring and evaluation } \\
\text { Mean of VIF }\end{array}$ & .611 & $\begin{array}{l}1.637 \\
\mathbf{1 . 6 8 4}\end{array}$ \\
\hline
\end{tabular}

a. Dependent Variable: Budget utilization

Source: field survey 2018, output generated by SPSS

As it is shown from the above table 9, the result shows that the mean of VIF 1.684 which is less than 10 and suggested that there is no severe multicollinearity problem in the regression model. And this briefly discussed that the multicolliniarity of this study shows that management support has the value of VIF 1.692 and tolerance value 0.591 , organizational work culture has the value of VIF 1.861 and tolerance value has 0.537 , employees related factor has the value of VIF 1.547 and tolerance value 0.646, and also monitoring and evaluation has the value of VIF 1.637 and tolerance value 0.611 . Generally the result showed that for all four factors are VIF for the variables have less than 10 and similarly the tolerance value greater than 0.1 . This revealed that there is no multicolliniarity problem between the independent variables in the regression model.

\subsubsection{Test of significance of individual explanatory variables}

In the parameter estimates table 10 below, see the coefficients, their standard errors, the Wald test, $\mathrm{df}(\mathrm{degree}$ of freedom) and associated p-values, the $95 \%$ confidence interval of the coefficients and odds ratios. Since p-values are less than alpha level (5\%) they are statistically significant; otherwise not significant. The thresholds are shown at the top of the parameter estimates output, and this indicates that the latent variable is cut to make the four groups that observed in data. The threshold coefficients are represented the intercepts, specifically the point in terms of a logit where budget utilization status might be predicted into the four categories. The number of coefficient was displayed one less than the number of categories of the variable. In this case, the coefficient is for the value of one and category three was the reference category and has a coefficient of zero.

To test for the significance of the regression coefficients, the null hypothesis $\left(\mathrm{H}_{\mathrm{o}}\right)$ and alternative hypothesis $\left(\mathrm{H}_{1}\right)$ are given by:
$\mathbf{H}_{\mathbf{0}}: \boldsymbol{\beta} \mathbf{j}=\mathbf{0}$
$\mathbf{H}_{1}: \boldsymbol{\beta} \mathbf{j} \neq \mathbf{0}$ 
Table 10: Parameter Estimates of Ordinal Logistic Regression

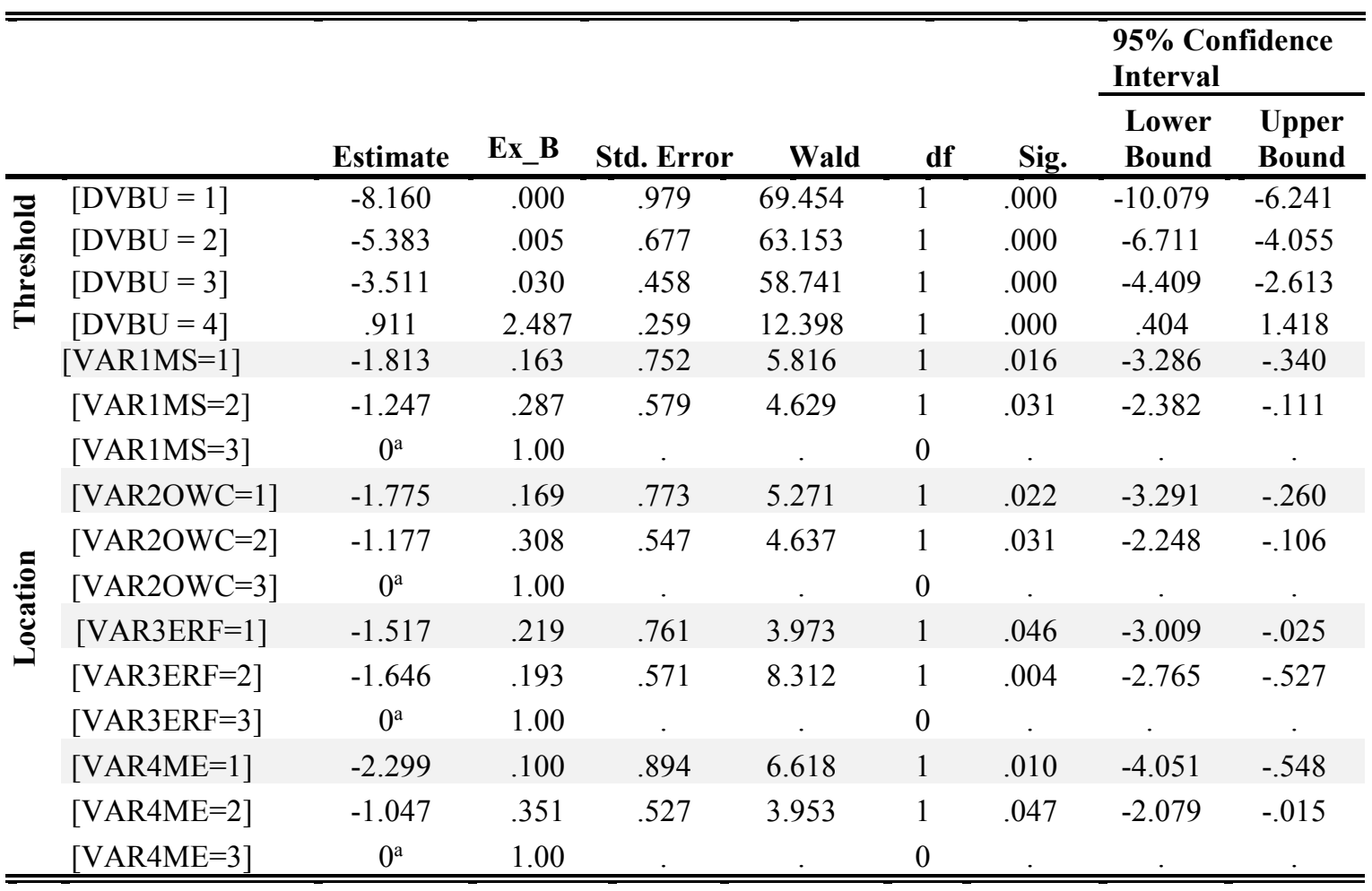

Link function: Logit.

a. This parameter is set to zero because it is redundant.

Where: DVBU=Dependent Variable, Budget utilization

$\mathrm{VAR}_{1} \mathrm{MS}=$ Variable 1, Management support

$\mathrm{VAR}_{2} \mathrm{OWC}=$ Variable 2, Organizational Work Culture

$\mathrm{VAR}_{3} \mathrm{ERF}=$ Variable 3, Employees Related Factor

$\mathrm{VAR}_{4} \mathrm{ME}=$ Variable 4, Monitoring and Evaluation

\subsection{Research hypothesis testing and interpret parameter estimates}

To achieve the objectives of the study and to test the related hypotheses the ordered logit model statistics computed in above table 10 were considered and demonstrated by parameter estimates the level of significance (sig-value) and odd ratio attained by each of the independent variables. The logistic regression results obtained from the model were utilized to test these hypotheses. The hypotheses sought to test for a significant influence of management support (MS), organizational work culture (OWC), employees related factor (ERF) and monitoring \& evaluation (ME) on the direct effect on budget utilization (BU). To see each hypothesis testing based on the logistic regression output on the above table. In the case of an ordinal outcome with three or more categories, the odd ratios for the logit model represents the odds of the higher category as compared to all lower categories combined. In other words, it is a cumulative odd ratio representing the increased likelihood to the next highest category relative to the lower categories for each unit increase in the predictor.

\subsubsection{Hypothesis one management support (VAR1)}

$\mathbf{H}_{1}$ : Management support has significant effect on budget utilization in BDCAHD.

The first hypothesis of this research proposed that management support. The result of logistic regression showed that in table 10 above, the individual test of this indicated that Wald test $=5.816$, p-value $=0.016, \operatorname{Exp} \_\beta=0.163$. The result showed that management support has effect on budget utilization as shown that Wald test of 5.816 which is different from zero and also significant in determining the outcome with the p-value 0.016 is less than significance level 0.05 . Then $\mathrm{H}_{0}$, null hypothesis was rejected at the 0.05 significant levels. Then as null hypothesis is rejected, the result showed that management support has significant effect on budget utilization in BDCAHD.

Hypothesis testing: to test the significance of the regression coefficients, the null and alternative hypotheses are given by:

$\mathrm{H}_{0}: \beta_{1}=0$

$\mathrm{H}_{1}: \beta_{1} \neq 0$, sig-value $=0.016<0.05$

Decision: As it can be seen in the table 10 above sig-value is less than 0.05 . Therefore, reject the null hypothesis, so there is significant non-linear relationship between budget utilization and management support. 
Interpretation: The independent variable, management support is significant at the $5 \%$ significance level of $\alpha$ or $95 \%$ confidence interval. Based on the above data concluded that, there is a significant relationship between management support and budget utilization within BDCAHD.

The odd of respondents disagreed on the statement management support has significant effect on budget utilization were 0.163 less than those who agreed on the statement, Keeping all other independent variables are constant.

\subsubsection{Hypothesis two organizational work culture (VAR2)}

$\mathbf{H}_{2}$ : Organizational work culture has significant effect on budget utilization in BDCAHD.

The result of logistic regression showed in table 10 above, that the individual test of organizational work culture indicated that Wald test $=5.271, p$-value $=.022$, Exp $\beta=0.169$. The result showed that organizational work culture has effect on budget utilization as revealed by Wald test of 5.271 which is different from zero and also significant in determining the outcome with the sig-value 0.022 is less than significance level 0.05 . Then $\mathrm{H}_{0}$, null hypothesis was rejected at the 0.05 significant levels. Then as null hypothesis is rejected, the result showed that organizational work culture has significant effect on budget utilization in BDCAHD.

Hypothesis testing: to test the significance of the regression coefficients, the null and alternative hypothesis is given by:

$\mathrm{H}_{0}: \beta_{2}=0$

$\mathrm{H}_{1}: \beta_{2} \neq 0$, sig-value $=0.022<0.05$

Decision: in the table above the p-value for the explanatory variable organizational work culture is less than 0.05 . Therefore, if it is significant, reject the null hypothesis of there is significant non-linear relationship between budget utilization and organizational work culture.

Interpretation: the explanatory variable, organizational work culture is significant at a given $5 \%$ significance level of $\alpha$ or $95 \%$ confidence interval. Based on the data indicated above it can be concluded that, there is a significant non-linear relationship between budget utilization by organizational work culture of the organization.

The odd of respondents disagreed on the statement organizational work culture has significant effect on budget utilization were 0.169 less than those who agreed on the statement. Keeping all other independent variables are constant.

\subsubsection{Hypothesis three employees related factor (VAR3)}

$\mathbf{H}_{3}$ : Employees related factor has significant effect on budget utilization in BDCAHD.

The result of logistic regression showed in table 10 above, that the individual test figures of employees related factor indicated by Wald test $=3.973, \mathrm{p}$-value $=.046, \operatorname{Exp} \beta=0.219$. The result showed that employees related factor has effects on budget utilization as revealed by Wald test of 3.973 which is different from zero and also significant in determining the outcome with the p-value 0.046 is less than significance level 0.05 . Then $\mathrm{H}_{0}$, null hypothesis was rejected at the 0.05 significant levels. Then as null hypothesis is rejected, the result showed that employees' related factor has significant effect on budget utilization in BDCAHD.

Hypothesis testing: to test the significance of the regression coefficient, improved accuracy the null and alternative hypothesis is given by:

$$
\begin{aligned}
& \mathrm{H}_{0}: \beta_{3}=0 \\
& \mathrm{H}_{1}: \beta_{3 \neq}, \text {, sig-value }=0.046<0.05
\end{aligned}
$$

Decision: As it can be seen in the table above sig-value of the explanatory variable, employees related factor is less than 0.05 . Therefore, reject the null hypothesis, there is significant non-leaner relationship between budget utilization and employees related factor.

Interpretation: the explanatory variable, employee's related factor is significant at the 5\% significance level of $\alpha$ or $95 \%$ confidence interval. Based on the above data indicated that it can be concluded that, there is a significant non-linear relationship between budget utilization and employees related factor.

The odd of respondents disagreed on the statement employees related factor has significant effect on budget utilization were 0.219 less than those who agreed on the statement. Keeping all other independent variables are constant.

\subsubsection{Hypothesis four monitoring and evaluation (VAR4)}

H4: Monitoring and evaluation have significant effect on budget utilization in BDCAHD.

The result of logistic regression showed in table 10 above, that the individual test figures of monitoring and evaluation indicated by Wald test $=6.618, p$-value $=.010, \operatorname{Exp} \beta=0.100$. The result showed that monitoring and evaluation has effects on budget utilization as showed by Wald test of 6.618 which is different from zero and also significant in determining the outcome with the p-value 0.010 is less than significance level 0.05 . Then $\mathrm{H}_{0}$, null hypothesis was rejected at the 0.05 significant levels. Then as null hypothesis is rejected, the result showed that monitoring \& evaluation have significant effect on budget utilization in BDCAHD.

Hypothesis testing: to test the significance of the regression coefficients, the null and alternative hypothesis is given by: 
$\mathrm{H}_{0}: \beta_{4}=0$

$\mathrm{H}_{1}: \beta_{4} \neq 0, p$-value $=0.010<0.05$

Decision: As it can be seen in the table above the p-value for the explanatory variable, monitoring and evaluation is less than 0.05 . Therefore, if it is significant at $5 \%$ significance level then, reject the null hypothesis of there is significant non-leaner relationship between budget utilization by monitoring and evaluation.

Interpretation: the qualitative explanatory variable, monitoring and evaluation is significant at a given $5 \%$ significance level of $\alpha$ or $95 \%$ confidence interval. Based on the above analysis it can be concluded that, there is a significant non-linear relationship between budget utilization by monitoring and evaluation of the organization.

The odd of respondents disagreed on the statement monitoring and evaluation has significant effect on budget utilization were 0.100 less than those who agreed on the statement. Keeping all other independent variables are constant.

8.7.5. Summary of hypothesis testing

In the hypothesis of four independent variables are statistically significant effects on dependent variable of budget utilization. This summary shows table 4.11 below.

Table 4. 11: Summary of hypothesis testing results

\begin{tabular}{|l|l|l|l|}
\hline H.No. & \multicolumn{1}{|c|}{ Hypothesis } & \multicolumn{1}{|c|}{$\begin{array}{l}\text { Results of } \\
\text { Finding }\end{array}$} \\
\hline $\mathrm{H}_{1}$ & Management support has significant effect on budget utilization. & 0.016 & Accepted \\
\hline $\mathrm{H}_{2}$ & Organizational work culture has significant effect on budget utilization. & 0.022 & Accepted \\
\hline $\mathrm{H}_{3}$ & Employees' related factor has significant effect on budget utilization. & 0.046 & Accepted \\
\hline $\mathrm{H}_{4}$ & Monitoring and evaluation have significant effect on budget utilization. & 0.010 & Accepted \\
\hline
\end{tabular}

Source: field survey 2018 , output generated by SPSS

\subsection{Regression Function of final model}

The function of logit typical application evenly distributed categories is calculated in the form of $\log \left(\frac{p i}{1-p i}\right)$.

The multiple non-linear logistic regression models are given as:

$$
\begin{aligned}
& \operatorname{logit}(P i)=\log \left(\frac{p i}{1-p i}\right)=\beta 0+\beta 1 X 1 i+\beta 2 X 2 i+\beta 3 X 3 i+\beta 4 X 4 i \\
& \log \left(\frac{p i}{1-p i}\right)=\beta 0+\beta 1 M S+\beta 2 O W C+\beta 3 E R F+\beta 4 M E \\
& =-8.160-1.813 \mathrm{MS}-1.7750 \mathrm{WC}-1.517 E R F-2.299 \mathrm{ME} \\
& =-5.383-1.813 \mathrm{MS}-1.775 \mathrm{OWC}-1.517 \mathrm{ERF}-2.299 \mathrm{ME} \\
& =-3.511-1.813 \mathrm{MS}-1.775 \mathrm{OWC}-1.517 E R F-2.299 \mathrm{ME} \\
& =0.911-1.81 \mathrm{MS}-1.775 \mathrm{OWC}-1.517 E R F-2.299 \mathrm{ME}
\end{aligned}
$$

Where: $p i=$ The probability that the event occurs

$\beta 0=$ is a constant, which is the value of dependent variable when all the independent variables are 0

$\mathrm{X}_{1} \mathrm{i}=\mathrm{MS}=$ Management support

$\mathrm{X}_{2} \mathrm{i}=\mathrm{OWC}=$ Organizational work culture

$\mathrm{X}_{3} \mathrm{i}=\mathrm{ERF}=$ Employees related factor

$\mathrm{X}_{4} \mathrm{i}=\mathrm{ME}=$ Monitoring and evaluation

$\beta 1, \beta 2, \beta 3, \beta 4=$ Regression coefficients of independent Variables or change induced by; MS, OWC, ERF, and ME.

\section{Conclusions}

The objective of this study was to investigate factors affecting budget utilization in case of BDCAHD, the research questions and objectives were answered. The major conclusion of the study results from inferential and descriptive analysis are presented as follows.

\subsection{Management support and budget utilization}

Based on the finding it has been concluded that, there is lack of management support in the department. And also, management support has significantly effect on budget utilization in case of BDCAHD.

\subsection{Organizational work culture and budget utilization}

Based on the finding it has been concluded that, there is lack of coordination, poor work culture and inefficient management system among the departments in the organization, which results in over and underutilization of budget. And also organizational work culture has significantly effect on budget utilization in case of BDCAHD. 


\subsection{Employees related factor and budget utilization}

Based on finding it has been concluded that, there is lack of experienced employee in the field to exercise the budget preparation and utilization activities properly. From the regression result of the study concludes that, employee related factor has significantly effect on budget utilization in case of BDCAHD.

\subsection{Monitoring and evaluation and budget utilization}

Based on finding it has been concluded that, there is lack of effective monitoring and evaluation in the health department. And also from the regression result it has been concluded that, monitoring \& evaluation have significantly effect on budget utilization in case of BDCAHD.

\section{Recommendations}

This section presents based on the results of the study obtained from findings and conclusions. In order to improve the efficiency of budget utilization of BDCAHD, and suggest possible recommendations to overcome such problems. Therefore, based on this research findings and conclusions the researcher forwards the following recommendations:

\subsection{Recommendations for practices}

10.1.1. Management support

Based on finding and conclusion, the study recommends that management should give attention for the attention of qualified employees, provide and facilitate short term training about budget utilization and budget issues, support each employee of public bodies' specifically in cash flow of management, plan and budget preparation, budget utilization and avoid procurement dalliance, adjust quarterly review meeting, and support participatory budget preparation, support to minimize the delay in allocation and approve the annual budget.

\subsubsection{Organizational work culture}

The study recommends that, there should be cooperation between budget, finance audit and procurement sections to improve level of budget utilization. Transparency and accountability should be exercised in health department and all ten health centers. Department of budget $\&$ finance standing committee should be adapt strong supervision and control mechanism. Reporting system should be effective on time, donor fund budget and government budget should equal attention for budget utilization in the organization.

\subsubsection{Employees related factor}

The study recommends that, management should give attention for the focus on qualified employees, the organization should also exercise job classification to give responsibilities for employees, and professionals should avoid careless behaviors and give attention to budget \& plan preparation and budget utilization. Enable the management to control employees with fail to perform their task properly. Training should arrange for employees to make them competent and to minimize knowledge gap about budget issues among professionals, and should improve service delivery system in the organization, and also arrange review meeting about plan and budget preparation, allocation and utilization.

\subsubsection{Monitoring and evaluation}

Based on finding and conclusion the study recommends that, the responsible bodies should implement continuous monitoring and evaluation of budget utilization. Internal and external auditors should timely and properly monitor and evaluate budget utilization in health department and health centers to create strong control mechanism and monitor budget transfers. And also give feedback about budget utilization to the responsible body on time.

Finally, the researcher also recommends that budget utilization should be improved, better budget plan, forecasting and focus on performance of government budget. Auditing is a series issue in department of health so; health department and all ten health centers should be audited on time by internal and external auditors.

\subsection{Recommendations for further Studies}

The following points are recommended that further researchers:

The result of this study demonstrated that there are other factors that significantly effect on budget utilization in case of BDCAHD and it is important to investigate such other factors in future researchers. Because of the limited time and resource, this study has been conducted in only one health department. However, this may not represent the situation of budget utilization across different areas and it is difficult to generalize at the country level. An interesting finding may come up by conducting studies at national or country wide by incorporating other independent variables that may determine budget utilization in public organizations. This study was modeled by the ordinal non-linear logistic regression model because of the study was used questionnaire by five point likert scale in categorical nature of the dependent variable and independent variables so, the researcher recommends future researchers to undertake the study by using other questionnaire method like linear regression model and other types of models to find other different findings. And also the study sought to investigate the factors affecting budget utilization in case of BDCAHD thus future researcher shall conduct research on other factors affect budget 
utilization and system develop of budget utilization.

\subsection{Contribution and implication of the study}

This study has its practical implications of the information affecting to various dimensions of budget utilization will be useful to BDCAHD to improve the area, which have managed to weak budget utilization. In health organization good service to give peoples were mandatory. This would encourage the government to improve effective utilization of budget. This would help in health department to look backward and forward to learn from failure and to decide on future activities. And also the information obtained regarding follow up and control mechanism, which is inferred, lack of monitoring and evaluation system, weak auditing system of ten health centers, weak management support and organizational work culture and also employees' related factor was effect on budget utilization.

\section{References}

Allen, R. and Tommasi, D., 2001. Multi-Year Budgeting and Investment Programming. 2001) Managing Government Expenditure: A Reference Book for Transition Countries. Paris: OECD SIGMA.

Ashebir Regassa(2017)public expenditure management with an emphasis on resource utilization, a case of Benishangul-Gumuz Regional State.

Geletaw Demera(2017) determinant of budget control in public organization in case of benishangul Gumuz Regional State

Ketema Muluneh (2015) assessment of budget preparation and utilization in case of Addis Ababa city administration health bureau.

Kothari, C.R. (2004). Research Methodology: Methods and Techniques. New Delhi, India: New Age International Publishers.

Mulugeta Dassa (2017) assessment of public budget allocation practice in case of BOFED of the SNNPRS.

Rebecca, S., Natasha, S. and Imran, A., 2011. A Guide to Public Financial Management Literature; For Practitioners in Developing Countries. Overseas Development Institute, December, London, http://www. who. int/pmnch/media/events/2013/pfm_literature. pdf (Erişim Tarihi: 3.11. 2014).

Silva, L.M.D. and Jayamaha, A.,2012. Budgetary process and organizational performance of apparel industry in Sri Lanka. Journal of emerging trends in economics and management sciences, 3(4), p.354.

Tilahun Bogale(2010) management and control special emphasis on ministry of national defence.

Tommasi, D., 2013. The budget execution process. In The international handbook of public financial management (pp. 285-311). Palgrave Macmillan, London.

Williams, J.J., Macintosh, N.B. and Moore, J.C., 1990. Budget-related behavior in public sector organizations: some empirical evidence. Accounting, Organizations and Society, 15(3), pp.221-246. 